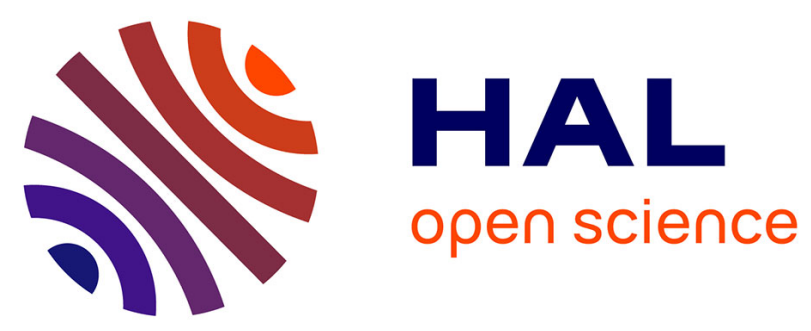

\title{
Measurements of the impedance matrix of a thermoacoustic core: Applications to the design of thermoacoustic engines
}

Flavio Bannwart, Guillaume Penelet, Pierrick Lotton, Jean-Pierre Dalmont

\section{- To cite this version:}

Flavio Bannwart, Guillaume Penelet, Pierrick Lotton, Jean-Pierre Dalmont. Measurements of the impedance matrix of a thermoacoustic core: Applications to the design of thermoacoustic engines. Journal of the Acoustical Society of America, 2013, 133 (5), pp.2650-2660. 10.1121/1.4796131 . hal02057382

HAL Id: hal-02057382

https://hal-univ-lemans.archives-ouvertes.fr/hal-02057382

Submitted on 5 Mar 2019

HAL is a multi-disciplinary open access archive for the deposit and dissemination of scientific research documents, whether they are published or not. The documents may come from teaching and research institutions in France or abroad, or from public or private research centers.
L'archive ouverte pluridisciplinaire HAL, est destinée au dépôt et à la diffusion de documents scientifiques de niveau recherche, publiés ou non, émanant des établissements d'enseignement et de recherche français ou étrangers, des laboratoires publics ou privés. 


\title{
Measurements of the impedance matrix of a thermoacoustic core: Applications to the design of thermoacoustic engines
}

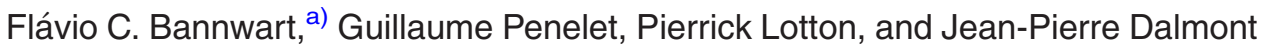 \\ Laboratoire d'Acoustique de l'Université du Maine, UMR CNRS 6613, Avenue Olivier Messiaen, \\ 72085 Le Mans Cedex 9, France
}

(Received 10 December 2012; revised 28 February 2013; accepted 8 March 2013)

\begin{abstract}
The successful design of a thermoacoustic engine depends on the appropriate description of the processes involved inside the thermoacoustic core (TAC). This is a difficult task when considering the complexity of both the heat transfer phenomena and the geometry of the porous material wherein the thermoacoustic amplification process occurs. An attempt to getting round this difficulty consists in measuring the TAC transfer matrix under various heating conditions, the measured transfer matrices being exploited afterward into analytical models describing the complete apparatus. In this paper, a method based on impedance measurements is put forward, which allows the accurate measurement of the TAC transfer matrix, contrarily to the classical two-load method. Four different materials are tested, each one playing as the porous element allotted inside the TAC, which is submitted to different temperature gradients to promote thermoacoustic amplification. The experimental results are applied to the modeling of basic standing-wave and traveling-wave engines, allowing the prediction of the engine operating frequency and thermoacoustic amplification gain, as well as the optimum choice of the components surrounding the TAC. (C) 2013 Acoustical Society of America. [http://dx.doi.org/10.1121/1.4796131]
\end{abstract}

PACS number(s): 43.35.Ud, 43.58.Bh [TB]

Pages: 2650-2660

\section{INTRODUCTION}

A thermoacoustic engine is an autonomous oscillator in which self-sustained acoustic waves are generated thanks to the external supply of heat. This device has been extensively studied in the past three decades because it can be used as a new kind of thermodynamic engine. ${ }^{1}$ The key element of a thermoacoustic engine is the so-called "thermoacoustic core" (TAC), which is the element in which the temperature field is heterogeneous. This TAC is surrounded by an acoustical network to compose a resonant device in which acoustic power is produced by thermoacoustic energy conversion. This acoustic power can then be supplied to an acoustic load, e.g., an electrodynamic alternator, as illustrated in Fig. 1. The TAC itself is divided into an "active" and a "passive" part. The "active" part consists of an open cell porous material (stack or regenerator) equipped with a hot (HHX) and an ambient (AHX) heat exchangers at its ends. These heat exchangers are used to maintain a steep temperature gradient, which is responsible for the onset of large amplitude acoustic waves oscillating at the frequency of the most unstable acoustic mode of the complete device. The "passive" part is the socalled thermal buffer tube (TBT), which refers to the waveguide region between the HHX and a secondary AHX. The open cell porous material can be any material with substantial surface area, high porosity, low thermal conductivity, and minimum flow resistivity. In the field of thermoacoustics, this

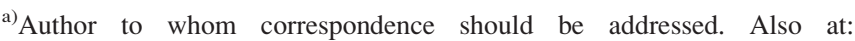
Universidade Estadual de Campinas, Faculdade de Engenharia Mecânica, Cidade Universitária “Zeferino Vaz," Barão Geraldo 13083-970 Campinas, São Paulo, Brazil. Electronic mail: fcbannwart@uol.com.br
}

element is referred to as "stack" or "regenerator." When employed as a stack in a standing-wave thermoacoustic engine, it has to ensure an imperfect heat transfer between the oscillating fluid and the adjacent solid walls (the nominal pore diameter must be on the order of magnitude of a few thermal acoustic boundary layer thicknesses). When employed as a "regenerator" in a traveling-wave thermoacoustic engine ${ }^{2}$ (also called thermoacoustic Stirling engine ${ }^{3}$ ), the pores must be sufficiently thin to ensure a quasi-isothermal contact between the solid walls and the oscillating fluid.

The design of thermoacoustic engines ${ }^{4}$ is partly based on empirical methods, mostly because the existing efficient prototypes include various elements like flow straighteners, tapered tubes, membranes or jet pumps, which are difficult to model accurately. Moreover, the marginal stability condition (that is the critical heat flux which has to be supplied to the hot heat exchanger to initiate self-sustained oscillations) is not easy to be determined because it requires an accurate knowledge of both the thermo-physical properties and the geometry of each element of the TAC. Indeed, when dealing with a regenerator or with a geometrically complicated stack, like a metallic foam, for example, it is not easy to describe acoustic propagation through such an anisotropic and tortuous material, and it is even more difficult to know the details of the temperature distribution ${ }^{5}$ under an assigned heat power input: this requires for instance to know the axial and transverse thermal conductivities of the material, and the heat-exchange coefficient between the material and the surrounding wall of the waveguide. Thus, an accurate description of a thermoacoustic engine not only requires to account for numerous nonlinear effects saturating wave amplitude growth, ${ }^{6-9}$ but it also involves an adequate modeling of heat transport within the TAC. 


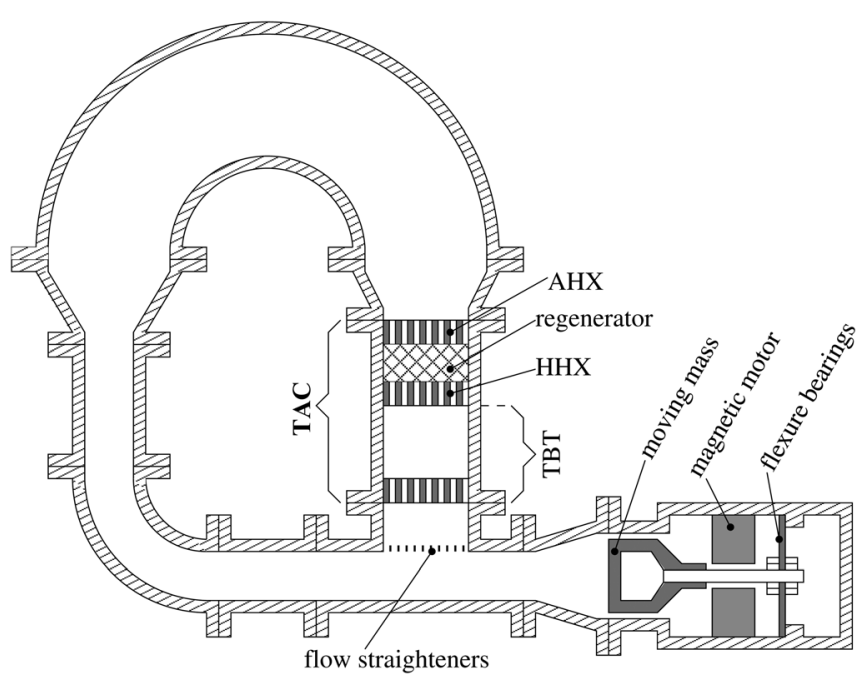

FIG. 1. Schematic view of a thermoacoustic engine. In this example, the acoustic load is a linear alternator.

An important research effort has been devoted to the description of acoustic propagation through stacks or regenerators within the framework of thermoacoustics, but most of these studies ${ }^{10-14}$ did not consider the existence of a temperature gradient, while the others ${ }^{15-18}$ did not treat the problem of heat transport within the TAC. More recently, alternative approaches have been proposed for the design of thermoacoustic engines, which amount to considering the TAC as a "black box" that can be characterized experimentally. Guedra et al. ${ }^{19}$ performed the measurement of the transfer matrices of a heated TAC by means of a classical "two load-four microphone" method. ${ }^{20,21}$ These transfer matrices were then introduced into an analytical model which predicts the onset of self-sustained oscillations in a thermoacoustic device. Experimental data obtained from a stack made up of a 600 cells per square inch (CPSI) ceramic catalyst have been introduced in the model and theoretical predictions of the onset conditions were consistent with those actually observed in different engines equipped with this TAC. However, as shown in this paper, the method developed by Guedra et al. fails to describe the behavior of a heated regenerator-based TAC.

In another context, Hatori et $a .^{22}$ have recently presented a similar approach to predict the operating point of a looped tube (equipped with a TAC) coupled to an acoustic load: the method consists in measuring the reflected acoustic impedance of both the looped tube (under various heating condition) and the acoustic load, so that the operating point (temperature gradient and working frequency) of the loaded thermoacoustic engine is obtained from the matching of the measured impedances.

This paper is a continuation of the works by Guedra et al. Its objective is actually twofold. First, it is to present an alternative method for the experimental characterization of a TAC, based on the accurate measurement of its impedance matrix by means of an acoustic impedance sensor. ${ }^{23,24}$ This method allows accurate descriptions of both a stackbased TAC and a regenerator-based TAC. Second, it is to use this method to perform measurements on different materials (Reticulated Vitreous Carbon foam, Nichrome foam, pile of stainless steel grids), and to use the experimental data for the optimum design of a thermoacoustic engine. In Sec. II, it is first shown that the two-load method used by Guedra et al. becomes inaccurate when the stack is replaced by a regenerator. Therefore, the alternative method mentioned above is presented, and it is shown that it succeeds on its purpose. In Sec. III A, a general modeling method is presented which predicts the working frequency and the thermoacoustic gain of a thermoacoustic engine equipped with the TAC characterized experimentally. This method is applied to the optimum design of simple standing wave and traveling wave engines using the different stack/regenerator materials mentioned above. Finally, a discussion is provided in Sec. IV, which aims at giving a conclusive perspective concerning the alternative method and also to emphasize the important features that should be addressed in the continuity of this study to improve the prediction of the performance of thermoacoustic engines.

\section{MEASUREMENT OF THE IMPEDANCE MATRIX OF THE THERMOACOUSTIC CORE}

\section{A. About the failure of the two-load method}

The thermoacoustic core under study is the one used by Guedra et al., ${ }^{19}$ except that the ceramic stack can be replaced by other materials. A scale plan of this TAC is presented in Fig. 2(a): its total length is $46-\mathrm{cm}$ with an inner radius of $16.9 \mathrm{~mm}$. The stack/regenerator is $7-\mathrm{cm} \mathrm{long}$, and it is bounded by the HHX and one of the AHX. The geometrical properties of the sample materials used in this study are reported in Table I. The AHX are made up of two copper pipes passing through a honeycombed aluminum disk, with flowing water inside the pipes at room temperature. The HHX is made up of a sample of ceramic catalyst (the same as the one described in Table I) in which a Nichrome resistance wire is coiled. The thickness of this sample is $1 \mathrm{~cm}$, and the heat resistance wire is connected to an electrical DC power supply controlling the heat power $Q_{H}$ dissipated by Joule effect throughout the wire.

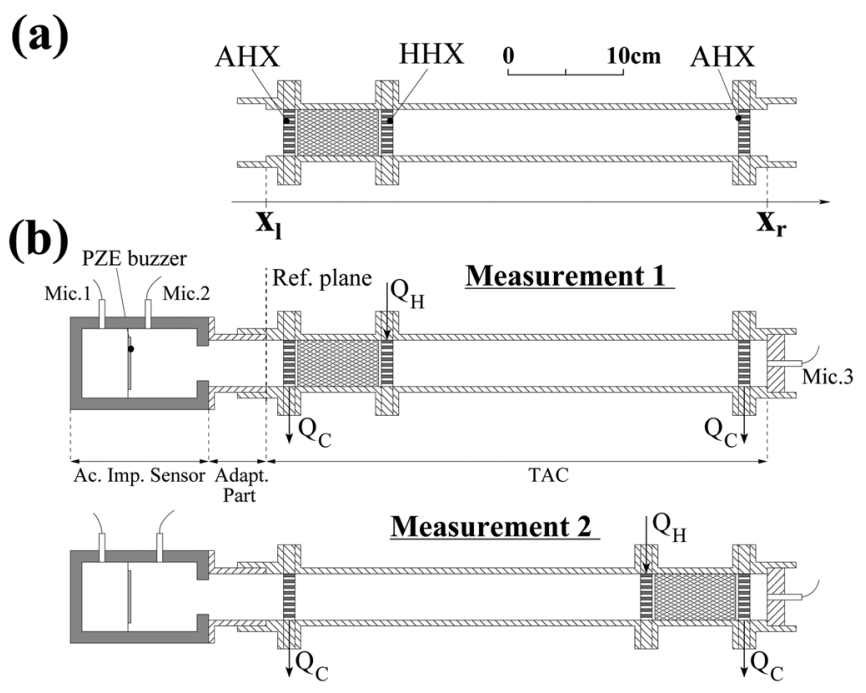

FIG. 2. (a) Sketch of the ThermoAcoustic Core, (b) Acoustic Impedance Sensor attached to the TAC in both directions. 
TABLE I. Geometrical properties of the sample materials.

\begin{tabular}{|c|c|}
\hline & Value \\
\hline \multicolumn{2}{|c|}{ Ceramic Catalyst } \\
\hline Porosity & $83 \%$ \\
\hline Number of cells per square inch & 600 CPSI \\
\hline \multicolumn{2}{|c|}{ Stainless Steel Grids ${ }^{\mathrm{a}}$} \\
\hline Porosity & $50 \%$ \\
\hline Wire mesh diameter & $0.08 \mathrm{~mm}$ \\
\hline Number of meshes per inch & 93.36 \\
\hline \multicolumn{2}{|c|}{ Nichrome Foam ${ }^{\mathrm{b}}$} \\
\hline Specific surface area & $2800 \mathrm{~m}^{2} / \mathrm{m}^{3}$ \\
\hline Average pore radius & $0.6 \mathrm{~mm}$ \\
\hline \multicolumn{2}{|c|}{ RVC Foam ${ }^{\mathrm{c}}$} \\
\hline Porosity & $96.5 \%$ \\
\hline Number of pores per inch & 100 PPI \\
\hline
\end{tabular}

${ }^{a}$ See http://www.gantois.fr, product ref. 104696.

${ }^{\mathrm{b}}$ See http://www.recemat.nl, product ref. RCM.NCAX.2733.

${ }^{\mathrm{c}} \mathrm{See}$ http://www.goodfellow.com.

In the following, our objective is to measure the transfer matrix of the thermoacoustic core as a function of both angular frequency $\omega$ and heat power supply $Q_{H}$. In order to do this, we first assume that harmonic plane waves are propagating in the thermoacoustic core at angular frequency $\omega$, so that the acoustic pressure $p(x, t)$ and acoustic volume velocity $u(x, t)$ are written as

$$
\zeta(x, t)=\Re\left[\tilde{\zeta}(x) e^{-j \omega t}\right],
$$

where $\zeta$ may be either $p$ or $u, \sim$ denotes the complex amplitude and $\Re()$ denotes the real part of a complex number. The transfer matrix $\mathbf{T}_{\mathrm{TAC}}\left(\omega, Q_{H}\right)$ is then defined as

$$
\left(\begin{array}{c}
\tilde{p}_{r} \\
\tilde{u}_{r}
\end{array}\right)=\left(\begin{array}{cc}
\mathcal{T}_{p p} & \mathcal{T}_{p u} \\
\mathcal{T}_{u p} & \mathcal{T}_{u u}
\end{array}\right)\left(\begin{array}{c}
\tilde{p}_{l} \\
\tilde{u}_{l}
\end{array}\right)=\mathbf{T}_{\mathrm{TAC}}\left(\begin{array}{c}
\tilde{p}_{l} \\
\tilde{u}_{l}
\end{array}\right)
$$

where $\tilde{p}_{l, r}=\tilde{p}\left(x_{l, r}\right)$ and $\tilde{u}_{l, r}=\tilde{u}\left(x_{l, r}\right)$ stand for the complex amplitudes of acoustic pressure $p$ and acoustic volume velocity $u$ at both ends of the TAC [see Fig. 2(a)].

The transfer matrices $\mathbf{T}_{\mathrm{TAC}}\left(\omega, Q_{H}\right)$ of different sample materials can be measured by means of a two-load method. ${ }^{19-21}$ The basic principles of this method consist in calculating $\tilde{p}_{l, r}$ and $\tilde{u}_{l, r}$ from the measurements of acoustic pressure by two pairs of microphones which are flushmounted on two straight ducts placed at each side of the TAC. The details of the two-load method applied to thermoacoustics can be found in Ref. 19. However, when pursuing the works mentioned above by trying to characterize new sample materials, we failed to measure accurately the T-matrix components for materials with low porosity, like stacked stainless steel grids for instance. In Fig. 3, the behavior of the $\mathcal{T}_{p p}$ $\left(\omega, Q_{H}\right)$ coefficient is presented as an arbitrary example, in the frequency range from 50 to $200 \mathrm{~Hz}$ (frequency step $=1 \mathrm{~Hz}$ ) and for 3 different values of heat power supply $Q_{H}$ that are $0 \mathrm{~W}, 36 \mathrm{~W}$, and $72 \mathrm{~W}$. The case of a ceramic stack is presented in Fig. 3, and the case of stacked stainless steel grids is presented in Fig. 4. From the comparison of Figs. 3 and 4,

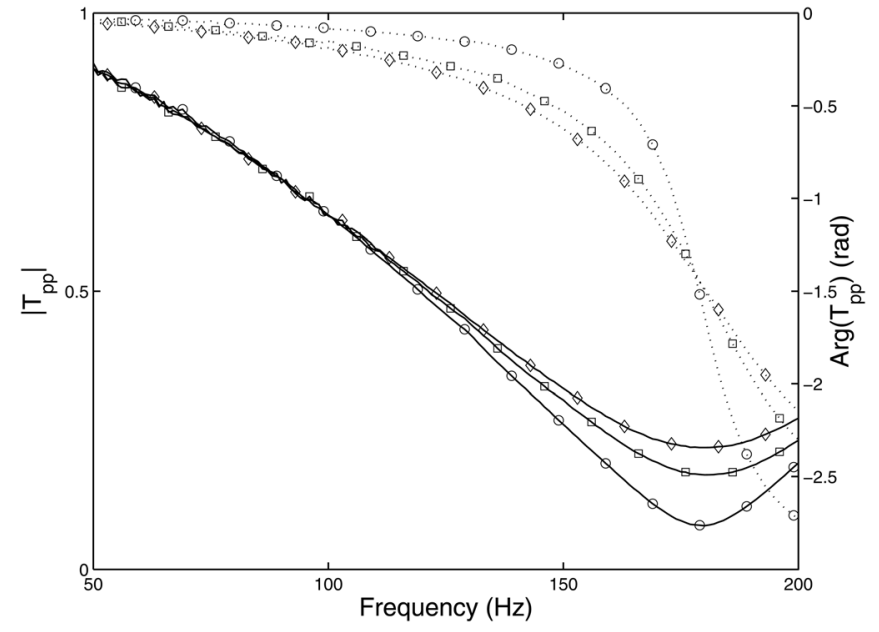

FIG. 3. Ceramic Catalyst: module (solid line) and phase (dotted line) of $\mathcal{T}_{p p}$ in the frequency domain for $Q_{H}=0 \mathrm{~W}$ (o), $Q_{H}=36 \mathrm{~W}$ (square), and $Q_{H}=72 \mathrm{~W}$ (diamond)-two-load method.

it appears clearly that the results obtained for the stainless steel grids are much more sensitive to noise. An objective indicator of the measurement accuracy can be obtained from the evaluation of the T-matrix reciprocity. When there is no heat supply into the system, it is expected the TAC to be reciprocal, meaning that the determinant of its T-matrix equals 1. The average value rc of reciprocity is defined as

$$
\mathbf{r c}=\frac{\sum_{i=1, \ldots, N} \mathcal{T}_{p p}\left(\omega_{i}, 0\right) \mathcal{T}_{u u}\left(\omega_{i}, 0\right)-\mathcal{T}_{p u}\left(\omega_{i}, 0\right) \mathcal{T}_{u p}\left(\omega_{i}, 0\right)}{N},
$$

where $\omega_{i}$ is the $i^{e s t}$ value of the operating frequency, and $N$ is the number of operating frequencies in the range of analysis. We also evaluated the standard deviation $\sigma_{\mathbf{r c}}$ of rc, which is an indicator of noise. In the case of the ceramic sample, the average value of reciprocity is $\mathbf{r c}=1.010+0.002 i$, hence very close to 1 (low bias level), and the standard deviation $\sigma_{\text {rc }}$ equals $0.002+0.002 i$ which is an indicator of low noise

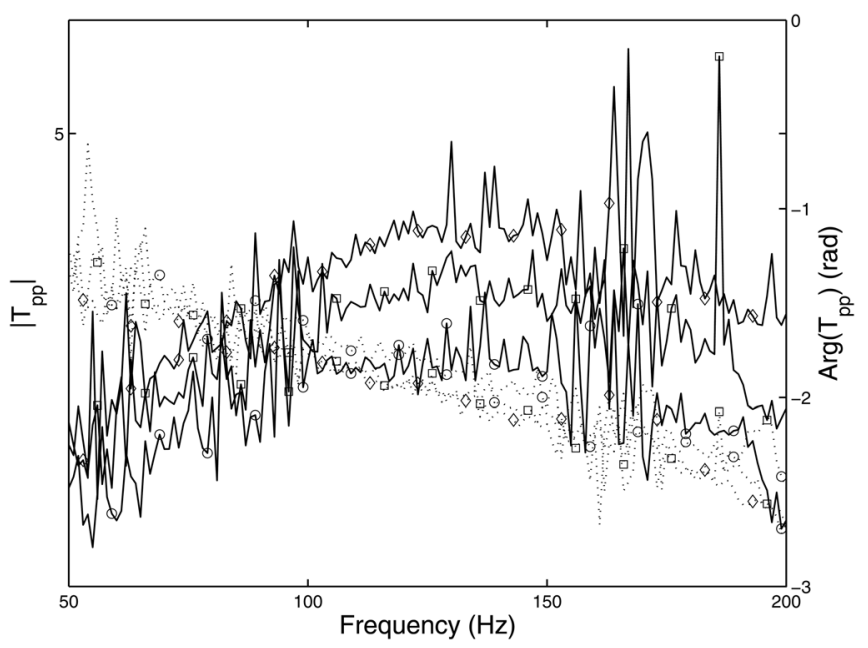

FIG. 4. Stainless Steel Grids: module (solid line) and phase (dotted line) of $\mathcal{T}_{p p}$ in the frequency domain for $Q_{H}=0 \mathrm{~W}(\mathrm{o}), Q_{H}=36 \mathrm{~W}$ (square), and $Q_{H}=72 \mathrm{~W}$ (diamond)—two-load method. 
level. However, in the case of the pile of stainless steel grids, one gets $\mathbf{r c}=0.996+0.005 i$ and $\sigma_{\mathbf{r c}}=0.134+0.123 i$ : even though very good on average, the standard deviation on reciprocity results very poor. It is a consequence of a high level of noise in $\mathbf{T}_{\mathrm{TAC}}$, in spite of applying the same wellcontrolled experimental procedures. This high level of noise is inherent to the application of the two-load method on a sample which has a low porosity: due to the high reflectivity of the material, the average level of acoustic pressure is much higher in the duct placed upstream from the sample than in the duct placed downstream from the sample, so that the microphones placed downstream are much more sensitive to noise. In principle, this kind of problem could be fixed by increasing the acoustic pressure level and the number of microphones, but this solution could lead to non-linear behavior and might increase the complexity of the experimental setup. Nevertheless, another way to overcome the limitation of this two-load method is to develop another method based on the measurement of the impedance matrix of the TAC. The impedance matrix of the TAC is defined as

$$
\left(\begin{array}{c}
\tilde{p}_{l} \\
\tilde{p}_{r}
\end{array}\right)=\left(\begin{array}{cc}
\mathcal{Z}_{11} & \mathcal{Z}_{12} \\
\mathcal{Z}_{21} & \mathcal{Z}_{22}
\end{array}\right)\left(\begin{array}{c}
\tilde{u}_{l} \\
\tilde{u}_{r}
\end{array}\right)=\mathbf{Z}_{\mathbf{T A C}}\left(\begin{array}{c}
\tilde{u}_{l} \\
\tilde{u}_{r}
\end{array}\right) .
$$

The transfer matrix coefficients, stated just as in the definition (2), are then calculated from the two-port equations that relate them to the $\mathrm{Z}$ matrix coefficients,

$$
\begin{aligned}
& \mathcal{T}_{p p}=\frac{\mathcal{Z}_{22}}{\mathcal{Z}_{12}}, \\
& \mathcal{T}_{p u}=\frac{\mathcal{Z}_{12} Z_{21}-Z_{22} Z_{11}}{\mathcal{Z}_{12}}, \\
& \mathcal{T}_{\text {up }}=\frac{1}{\mathcal{Z}_{12}}, \\
& \mathcal{T}_{\text {uи }}=\frac{-\mathcal{Z}_{11}}{\mathcal{Z}_{12}} .
\end{aligned}
$$

\section{B. Measurement of the impedance matrix}

The impedance matrix coefficients are actually measured by means of an acoustic impedance sensor ${ }^{25}$ coupled to the TAC under test. This alternative method, presented hereafter, is less sensitive to noise than the two-load method. In addition, it is more direct than the two-load method since there is no need to account for plane wave propagation in some ducts surrounding the TAC, and there is no limitations in the frequency range of analysis relative to the distance between microphones. ${ }^{20}$

A schematic drawing of the experimental apparatus used for the determination of the impedance matrix of a thermoacoustic core is shown in Fig. 2(b). The acoustic impedance sensor itself consists of a piezoelectric buzzer loaded by a small cavity on its rear face and radiating toward a short duct on its front face. Two microphones (labeled Mic 1 and Mic 2) are flush-mounted along the wall at both sides of the piezoelectric buzzer. The front cavity of the impedance sensor is coupled to one end of the TAC under test. The other end of the TAC is closed by a rigid wall. Mic 1 measures the pressure $p_{1}(t)$ inside the rear cavity, while Mic 2 measures the pressure $p_{2}(t)$ in the front duct. A third microphone (labeled Mic 3), flush-mounted in the center of the closing rigid wall, measures the pressure $p_{3}(t)$ at the end of the TAC. At low frequencies, below the first resonance of the rear cavity, the pressure is uniform in the cavity and $p_{1}(t)$ is proportional to the volume velocity $u(t)$ produced by the buzzer. Then, from the measurement of pressures $p_{1}(t)$ and $p_{2}(t)$, and taking into account the acoustic propagation along the front duct, the pressure $\tilde{p}_{l}$ and the volume velocity $\tilde{u}_{l}$ can be calculated in the reference plane $x=x_{l}$ at the entrance of the thermoacoustic core, leading to the estimation of the input acoustic impedance $\mathcal{Z}_{11}=\tilde{p}_{l} / \tilde{u}_{l}$. Besides, the transfer impedance $\mathcal{Z}_{21}=\tilde{p}_{r} / \tilde{u}_{l}$ is obtained from the measurement of $p_{3}(t)=p\left(x_{r}, t\right)$. Due to the asymmetry of the TAC, a second measurement is needed in order to fulfill the four coefficients of the impedance matrix. The two last coefficients $\mathcal{Z}_{22}$ and $\mathcal{Z}_{12}$ of the impedance matrix are measured in the same way when inverting the impedance sensor and the closing rigid wall as shown in Fig. 2(c).

In order to account for the microphone positions relative to the reference plane, the Z-matrix coefficients $\mathcal{Z}_{11}$ and $\mathcal{Z}_{21}$ are generally expressed as follow: ${ }^{26}$

$$
\begin{aligned}
& \mathcal{Z}_{11}=\frac{\left(H_{21} / K\right)-\beta}{1-\left(\delta H_{21} / K\right)}, \\
& \mathcal{Z}_{21}=\frac{H_{31}\left(1+\delta \mathcal{Z}_{11}\right)}{\delta K_{T}},
\end{aligned}
$$

where the terms

$$
H_{i j}=\frac{\tilde{p}_{i} \tilde{S}_{i}}{\tilde{p}_{j} \tilde{S}_{j}}
$$

are the transfer functions that relate the sound pressure measurements, and where $\tilde{S}_{i}$ refers to the sensitivity of Mic $i$. The terms $\beta$ and $\delta$ in Eqs. (9) and (10) are parameters related to the geometry, which are assumed to be known, while $K$ and $K_{T}$ are related to the calibration of the device. ${ }^{26}$ For the experimental determination of $\mathcal{Z}_{22}$ and $\mathcal{Z}_{12}$, the same Eqs. (9) and (10) are used, respectively, when inverting the TAC. The calibration process is, at first, to remove the TAC and close the acoustic impedance sensor by the rigid plate that supports microphone 3. The transfer functions $H_{21}$ and $H_{31}$ are then measured and here labeled with the subscript "cal." As a consequence of such a configuration, the impedance at the reference plane is the same as the transfer impedance to the microphone 3 due to the fact that the position is coincident. Assuming $1 / Z_{11, \text { cal }}=1 / Z_{21, \text { cal }} \approx 0$ leads to $K=\delta H_{21, \text { cal }}$ and $K_{T}=H_{31, \text { cal }}$ (see Ref. 26 for more details). Therefore, as a final result of the calibration, the parameters $K$ and $K_{T}$ are obtained from the measurements of $H_{21, \text { cal }}$ and $H_{31, \text { cal }}$ and both ratios of sensitivity $\tilde{S}_{2} / \tilde{S}_{1}$ and $\tilde{S}_{3} / \tilde{S}_{1}$ are implicitly taken into account. Once such parameters are available and the calibration transfer functions are registered, the following step is enabled, which concerns the impedance measurement itself. 
Special cares must be taken when making the experiments, like ordinary precautions against acoustic leakage and, first of all, a close monitoring over the stabilization of the temperature distribution along the TAC. Indeed the variation of temperature distribution has strong impact on the resulting two-port coefficients. Therefore, the steady state temperature distribution is assumed to be reached after a time delay, which is experimentally evaluated by analyzing the time evolution of the acoustic pressure transfer functions $H_{21}$ and $H_{31}$ when changing the level of heat power supply. In our experiment, this time delay is around $13 \mathrm{~min}$. The experimental procedure for the impedance measurements is led, hence, increasing the heat power supply from 0 up to $81 \mathrm{~W}$ by steps of $9 \mathrm{~W}$. The measurements are made for each heat condition, one by one, and the whole sequence is followed in just one direction [see Fig. 2(b), "measurement 1"] till its end. Afterward, the heat power supply is turned off. The TAC is then naturally cooled and it reaches the ambient temperature homogeneously after a few hours. Subsequently, the impedance sensor is inverted [see Fig. 2(b), "measurement 2"], and the same sequence of measurements is applied, following the same rising heat supplies and keeping the same time intervals between them, in order to obtain a thermal field inside the TAC as similar as possible to the one obtained in the previous set of measurements. Nevertheless, this procedure may be sensitive to ambient temperature variations, or even pressure variations, as the total duration of both sequences of measurements takes several hours. But proceeding this way led us to the best results. As explained before, the acoustic impedance sensor allows measurements in a much wider frequency range than the two-load method does. Hence, we chose to investigate the problem within the interval between 30 to $500 \mathrm{~Hz}$, by steps of $1 \mathrm{~Hz}$. This allows investigation of a wide range of operating frequencies for different engines configurations. However, in order to compare our results with those of the two-load method, we chose in the following to show the two-port coefficients for a narrower interval, between 50 and $200 \mathrm{~Hz}$.

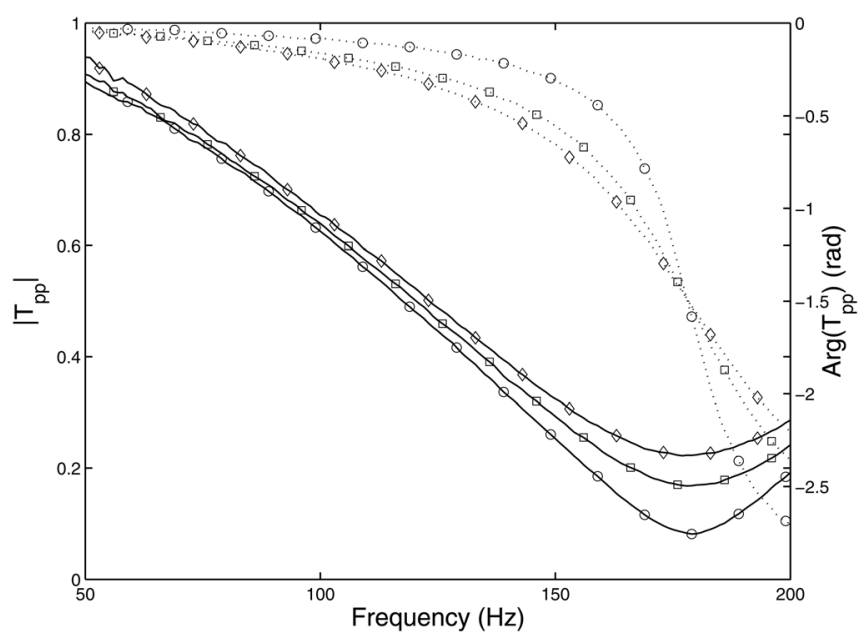

FIG. 5. Ceramic Catalyst: module (solid line) and phase (dotted line) of $\mathcal{T}_{p p}$ in the frequency domain for $Q_{H}=0 \mathrm{~W}(\mathrm{o}), Q_{H}=36 \mathrm{~W}$ (square), and $Q_{H}=72 \mathrm{~W}$ (diamond) - Alternative method.

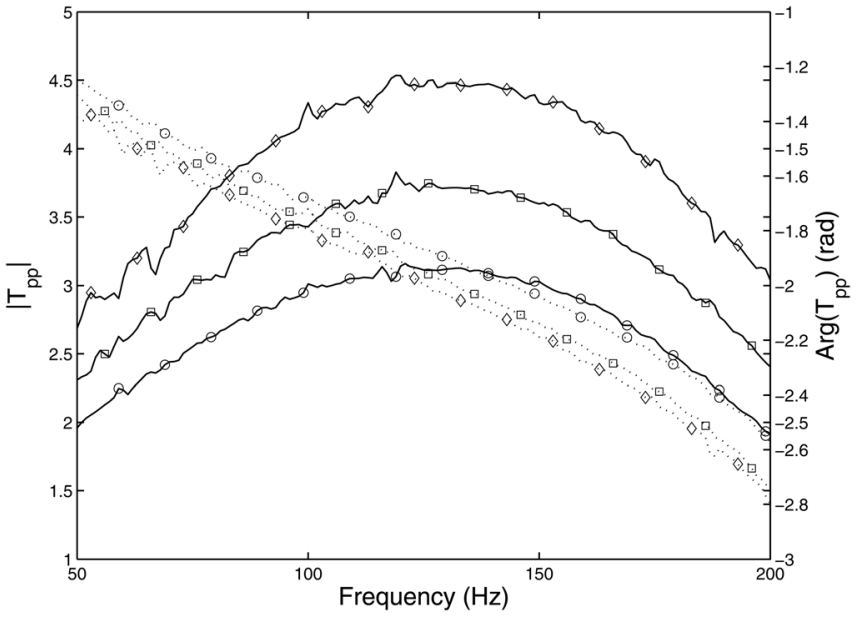

FIG. 6. Stainless Steel Grids: module (solid line) and phase (dotted line) of $\mathcal{T}_{p p}$ in the frequency domain for $Q_{H}=0 \mathrm{~W}(\mathrm{o}), Q_{H}=36 \mathrm{~W}$ (square), and $Q_{H}=72 \mathrm{~W}$ (diamond)-Alternative method.

\section{Experimental results}

The impedance matrix coefficients measured with this method can be used to evaluate the transfer matrix coefficients thanks to the relation (5). As an example, the behavior of the $\mathcal{T}_{p p}\left(\omega, Q_{H}\right)$ coefficient, calculated from the impedance matrix measurement, is presented in Figs. 5 and 6 for the same experimental configuration as the one used for Fig. 3.

From the comparison of Fig. 3 and Fig. 5, one can see that the two measurement methods lead to almost similar results in the case of the ceramic stack. When analyzing the no-heat input condition $\left(Q_{H}=0 \mathrm{~W}\right)$, good outcomes are achieved with the alternative method, either for the average value of reciprocity, resulting in $\mathbf{r c}=1.002-0.001 i$ (low bias level), or for the standard deviation, resulting in $\sigma_{\text {rc }}=0.001+0.002 i$ (low noise level).

From the comparison of Fig. 4 and Fig. 6, it is clear that the results obtained with the alternative method for the regenerator made of stainless steel grids are much less sensitive to noise than the ones obtained with the two-load method. Indeed, for $Q_{H}=0 \mathrm{~W}$, the indicator reciprocity reaches an average value of $\mathbf{r c}=1.004+0.009 i$ (low bias level) and a standard deviation of $\sigma_{\text {rc }}=0.008+0.007 i$ (low noise level).

Thus, since it has been shown that the alternative method presented in this paper lead to good results for both stack and regenerator, it can be used to characterize other materials which can be anticipated to behave in between "stack" and "regenerator." This has been successfully done for the $\mathrm{NiCr}$ Foam and the RVC Foam in the frame of this work. As an indicator of the measurement quality, the average value of reciprocity and the corresponding standard deviation for $Q_{H}=0 \mathrm{~W}$ are given in Table II for these materials. Low bias and low noise level are both achieved as well.

\section{APPLICATION TO THE DESIGN OF A THERMOACOUSTIC ENGINE}

The knowledge of the T-matrix coefficients of the TAC at different heating conditions can be used to predict both the heat power supply and the operating frequency which correspond to the onset of self-sustained acoustic waves in a 
TABLE II. Average reciprocities and standard deviations.

\begin{tabular}{llcc}
\hline \hline & Variable & Two-Load & Z-matrix \\
\hline Ceramic Catalyst & rc & $1.010+0.002 i$ & $1.002-0.001 i$ \\
& $\sigma_{\text {rc }}$ & $0.002+0.002 i$ & $0.001+0.002 i$ \\
Stainless Steel Grids & rc & $0.996+0.005 i$ & $1.004+0.009 i$ \\
& $\sigma_{\text {rc }}$ & $0.134+0.123 i$ & $0.008+0.007 i$ \\
RVC Foam & rc & - & $1.000-0.002 i$ \\
& $\sigma_{\text {rc }}$ & - & $0.002+0.002 i$ \\
NiCr Foam & rc & - & $1.001-0.000 i$ \\
& $\sigma_{\text {rc }}$ & - & $0.002+0.001 i$ \\
\hline \hline
\end{tabular}

thermoacoustic engine equipped with the TAC characterized beforehand, as done by Guedra et al. ${ }^{19}$ It is, however, worth mentioning that such a task requires having made the $\mathrm{T}$ matrix measurements for numerous values of heat power supply $Q_{H}$, since the accuracy of the predictions of onset is directly related to the step of heat supply $\Delta Q$ between two set of measurements. An alternative but similar approach consists in developing an adequate modeling which would predict both the operating frequency $f_{o p}$. and the intrinsic thermoacoustic amplification gain $G$ associated both to a given heat power $Q_{H}$ supplied to the TAC and to the respective geometry of each element surrounding this TAC. In the following, we present a general modeling method based on this alternative approach, which allows for the calculation of both the operating frequency $f_{o p}$. and the thermoacoustic gain $G$ in a thermoacoustic engine from the measured T-matrix components of a given TAC under a given heat power supply $Q_{H}$. Note that the two parameters $f_{o p}$. and $G$ also depend on the dimensions of the waveguides connected to the TAC, which notably determine the spatial distribution of the acoustic field in the complete device. Therefore, an optimization of the geometric parameters of each element surrounding the TAC can be then performed by searching the geometrical configuration leading to a maximum value of the gain $G$. Thereby, this modeling method allows us to compare the potential performances of the different sample materials characterized in Sec. II C, taking $G$ as the main comparative parameter. This method is applied in the following to the optimization of the performances of a simple standing-wave and a simple closed-loop engine (see Fig. 7), but it is also easily applicable to a more complicated engine as the one of Fig. 1.

\section{A. Theory}

Let us consider the simple thermoacoustic engines which are depicted in Fig. 7. The first one is a standing-wave engine which basically consists of the TAC surrounded by two straight ducts closed by rigid ends. The second one is made of a closed-loop, containing the TAC, connected to a straight duct. Note that in the configuration of the closedloop engine, it is considered here that the curvature of the waveguide, and especially that of the TAC, has no impact on the propagation of acoustic waves.

\section{Standing-wave engine}

Let us consider at first the standing wave engine of Fig. 7. Two straight ducts of lengths $L_{1}$ and $L_{2}$ are connected to both sides of the TAC. These ducts have the same inner diameter than the TAC. Assuming the propagation of plane acoustic waves at angular frequency $\omega$, it is possible to relate the acoustic pressure $\tilde{p}_{L}$ and volume velocity $\tilde{u}_{L}$ at position $x=L$ to the pressure $\tilde{p}_{0}$ and volume velocity $\tilde{u}_{0}$ at position $x=0$ as follows:

$$
\left(\begin{array}{c}
\tilde{p}_{L} \\
\tilde{u}_{L}
\end{array}\right)=\mathbf{T}_{\mathbf{2}} \cdot \mathbf{T}_{\mathbf{T A C}} \cdot \mathbf{T}_{\mathbf{1}} \cdot\left(\begin{array}{c}
\tilde{p}_{0} \\
\tilde{u}_{0}
\end{array}\right)=\mathbf{T}_{\mathbf{t o t}} \cdot\left(\begin{array}{c}
\tilde{p}_{0} \\
\tilde{u}_{0}
\end{array}\right),
$$

where the T-matrices

$$
\mathbf{T}_{\mathbf{j}_{(\mathrm{j}=\mathbf{1}, 2)}}=\left[\begin{array}{lc}
\cos \left(k L_{j}\right) & i Z_{c} \sin \left(k L_{j}\right) \\
\frac{i}{Z_{c}} \sin \left(k L_{j}\right) & \cos \left(k L_{j}\right)
\end{array}\right]
$$

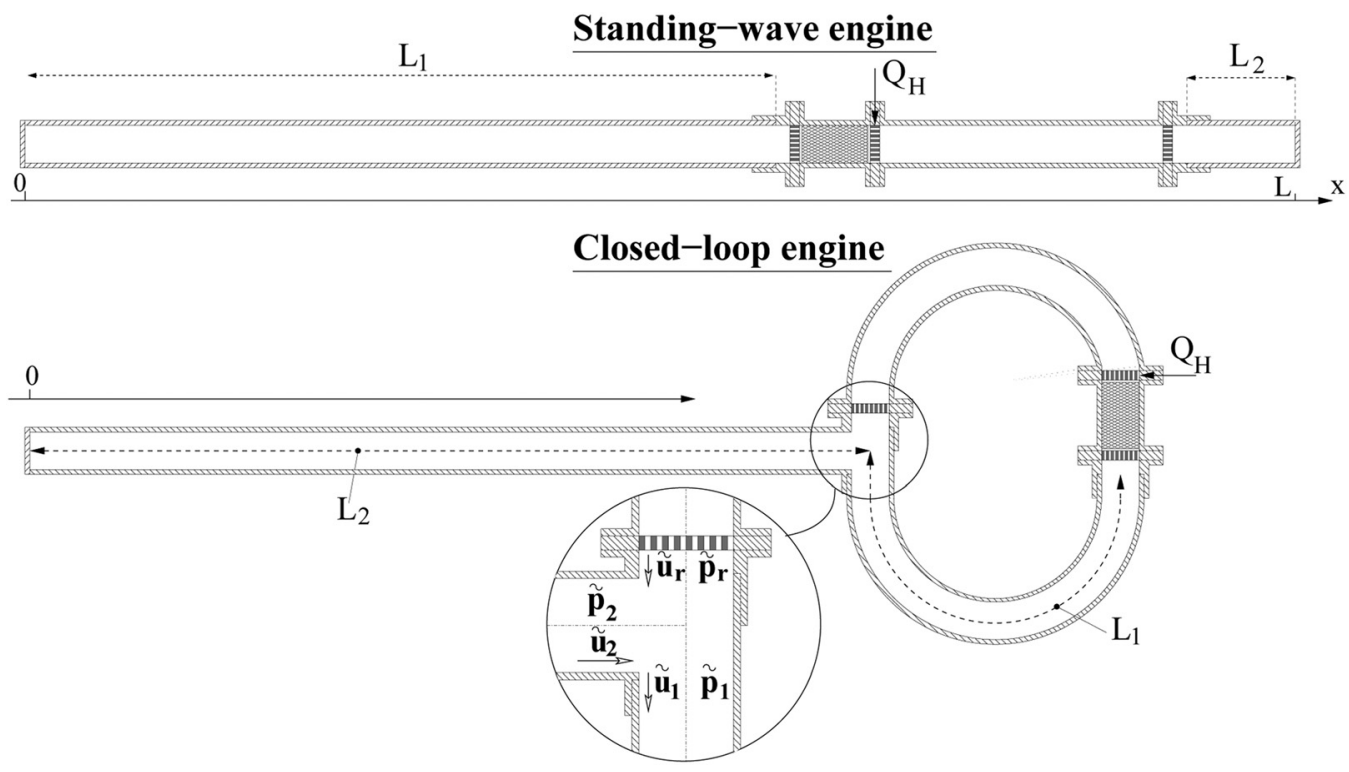

FIG. 7. Basic configurations for standing-wave and closed-loop engines. 
characterize the lossy propagation of acoustic waves in the ducts of respective lengths $L_{1}$ and $L_{2}$ and where

$$
k=\frac{\omega}{c_{0}}\left[1+\frac{f_{\nu}+(\gamma-1) f_{\kappa}}{1-f_{\nu}}\right]^{1 / 2}
$$

and

$$
Z_{c}=\frac{\rho_{0} c_{0}}{S\left\{\left(1-f_{\nu}\right)\left[1+(\gamma-1) f_{\kappa}\right]\right\}^{1 / 2}}
$$

are the complex wave number and the characteristic impedance of the ducts, respectively. In Eqs. (14) and (15), $\rho_{0}$ is the fluid density at room temperature, $c_{0}$ is the adiabatic sound speed, $\gamma$ is the specific heat ratio of the fluid, $S$ is the duct cross-sectional area, and the functions $f_{\kappa}$ and $f_{\nu}$ characterize the thermal and viscous coupling between the oscillating fluid and the duct walls. ${ }^{1,15}$ Then, taking into account that $\tilde{u}_{L}=0$ (rigid termination), one gets

$$
\left(\begin{array}{c}
\tilde{p}_{0} \\
\tilde{u}_{0}
\end{array}\right)=T_{\text {tot }}^{-1}\left(\begin{array}{l}
\tilde{p}_{L} \\
0
\end{array}\right)=\left(\begin{array}{cc}
A_{p p} & A_{p u} \\
A_{\text {up }} & A_{\text {uи }}
\end{array}\right)\left(\begin{array}{l}
\tilde{p}_{L} \\
0
\end{array}\right),
$$

from which one can write the reflected acoustic impedance at position $x=0$,

$$
Z_{0}=\frac{\tilde{p}_{0}}{\tilde{u}_{0}}=\frac{A_{p p}}{A_{u p}} .
$$

The knowledge of this reflected acoustic impedance $Z_{0}$ $=Z_{0}\left(L_{1}, L_{2}, Q_{H}, \omega\right)$ can be used to determine the resonance frequencies of the complete device. In particular, if the left side of the engine is also terminated by a rigid plug (infinite impedance), then the principle of impedance matching tells that the resonance frequencies correspond to the maxima of $\left|Z_{0}\right|$ (these maxima should tend toward infinity if losses were neglected). As an illustrative example, the modulus of $Z_{0}$ and its real part are presented for three levels of heat supply
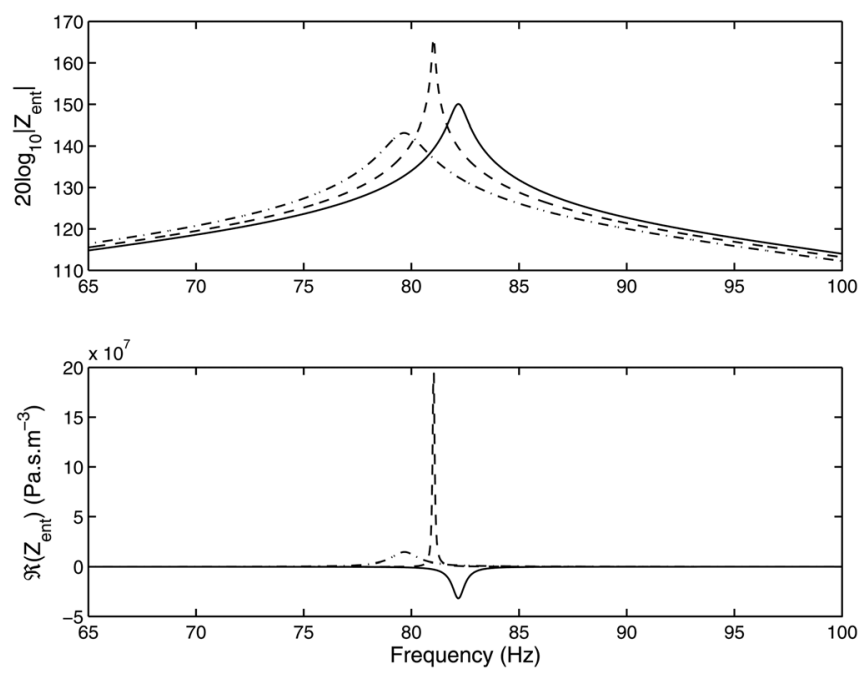

FIG. 8. Ceramic Catalyst: modulus and real part of the reflected acoustic impedance for $Q_{H}=9 \mathrm{~W}$ (dash-dotted line), $Q_{H}=18 \mathrm{~W}$ (dashed line), and $Q_{H}=27 \mathrm{~W}$ (solid line). in Fig. 8 as a function of the frequency in the particular case when $L_{1}$ and $L_{2}$ are fixed to $160 \mathrm{~cm}$ and $10 \mathrm{~cm}$, respectively. The reflected impedance $Z_{0}$ is calculated using the experimental transfer matrix $\mathbf{T}_{\mathbf{T A C}}$ of the ceramic stack previously determined under different levels of heat power supply.

From the analysis of $\left|Z_{0}\right|$, it appears that the first resonance frequency $f_{o p}$. of the system is around $80 \mathrm{~Hz}$, and that it tends to increase with heating. Moreover, the quality factor of the resonance (which is inversely proportional to the width of the resonance peak) increases as $Q_{H}$ increases from 9 to $18 \mathrm{~W}$. This is consistent with our expectations since the quality factor should tend toward infinity as the system approaches the threshold of thermoacoustic instability. ${ }^{27}$ However, the width of the resonance peak increases as $Q_{H}$ increases from 18 to $27 \mathrm{~W}$, which seems surprising at first sight. Actually, this means that the system is above threshold for $Q_{H}=27 \mathrm{~W}$ so that the width of the resonance peak is no longer related to the quality factor of the resonance: this quality factor should become negative through onset, ${ }^{28}$ for which the net attenuation of sound becomes negative. Additional insight arises from the analysis of $\Re\left(Z_{0}\right)$ at resonant frequency $f_{\text {op. }}$, leading to the same conclusions: if $Q_{H} \leq 18 \mathrm{~W}$, the real part of $Z_{0}\left(f_{o p}\right)$ is positive which means that work is absorbed by the device, but for $Q_{H}=27 \mathrm{~W}$ the real part of $Z_{0}$ becomes negative which means that work is produced by the system. ${ }^{22}$ In other words, if $Q_{H}=27 \mathrm{~W}$ the system is above threshold, and self-sustained acoustic waves should oscillate at frequency $f_{o p}$. (in as much as it is considered that the generation of large amplitude acoustic oscillations does not induce variations of the temperature field inside the TAC with subsequent variations of its transfer matrix).

Once the reflected impedance $Z_{0}$ is determined, the resonance frequencies of the device are known. It is then possible to choose a particular working frequency and to calculate a thermoacoustic amplification gain associated to the acoustic mode under consideration. Let us assume that the operating angular frequency $\omega_{o p}$. is fixed to that of the first acoustic mode (which is generally the most unstable mode). Let us assume also that the acoustic pressure $\tilde{p}_{0}$ at position $x=0$ is fixed to some arbitrary value $P_{0}$, from which one easily gets the corresponding volume velocity $\tilde{u}_{0}=P_{0} / Z_{0}$, together with the acoustic pressures and volume velocities at positions $x_{l}, x_{r}$, and $L$ (which are deduced from $\tilde{u}_{0}, \tilde{p}_{0}$, and the T-matrices $\mathbf{T}_{\mathbf{1}}, \mathbf{T}_{\mathbf{T A C}}$, and $\mathbf{T}_{\mathbf{2}}$ ). Therefore, it is possible to calculate the acoustic power

$$
\begin{aligned}
\mathbf{W}_{\text {loss }}= & {\left[\frac{1}{2} \Re\left(\tilde{p}_{l} \tilde{u}_{l}^{*}\right)-\frac{1}{2} \Re\left(\tilde{p}_{0} \tilde{u}_{0}^{*}\right)\right] } \\
& +\left[\frac{1}{2} \Re\left(\tilde{p}_{L} \tilde{u}_{L}^{*}\right)-\frac{1}{2} \Re\left(\tilde{p}_{r} \tilde{u}_{r}^{*}\right)\right]
\end{aligned}
$$

dissipated by viscous and thermal losses into the ducts, where $^{*}$ denotes the conjugate of a complex number, and to calculate the acoustic power

$$
\mathbf{W}_{\mathbf{T A C}}=\left[\frac{1}{2} \Re\left(\tilde{p}_{r} \tilde{u}_{r}^{*}\right)-\frac{1}{2} \Re\left(\tilde{p}_{l} \tilde{u}_{l}^{*}\right)\right]
$$


produced within the TAC. The powers $\mathbf{W}_{\text {loss }}$ and $\mathbf{W}_{\text {TAC }}$ depend on the unknown parameter $P_{0}$, but their ratio does not. Then, a thermoacoustic amplification gain $G$ can be defined as follows:

$$
G=\frac{\mathbf{W}_{\mathrm{TAC}}}{\left|\mathbf{W}_{\text {loss }}\right|} .
$$

This parameter $G$ has the meaning of an energy balance in the system: if $G>1$, then the acoustic work produced by thermoacoustic effect in the TAC is higher than the losses in the remaining of the device, so that the extra work can be provided to an acoustic load.

The results obtained for the frequency of operation $f_{o p}$. and the corresponding thermoacoustic amplification gain $G$ are reported in Table III: the engine under consideration is a standing wave engine equipped with a ceramic catalyst stack, while $L_{1}$ and $L_{2}$ are fixed to $160 \mathrm{~cm}$ and $10 \mathrm{~cm}$, respectively. The calculations of $G$ are consistent with the conclusion mentioned above that the heat supply corresponding to onset threshold $(G=1)$ is between 18 and $27 \mathrm{~W}$.

\section{Closed-loop engine}

The same kind of analysis than the one presented above can be realized in the case of a closed-loop engine (see Fig. 7) which is the basic geometrical configuration encountered in thermoacoustic Stirling engines that use a regenerator instead of a stack. As in Sec. III A 1, the first step consists in determining the reflected impedance $Z_{0}$ from the T-matrices $\mathbf{T}_{\mathbf{1 , 2}}$ and $\mathbf{T}_{\text {TAC. }}$. After a few calculations, one gets

$$
Z_{0}=\frac{\mathcal{B}_{p p} Z_{2}+\mathcal{B}_{p u}}{\mathcal{B}_{u p} Z_{2}+\mathcal{B}_{u u}}
$$

with

$$
\begin{aligned}
& \left(\begin{array}{ll}
\mathcal{B}_{p p} & \mathcal{B}_{p u} \\
\mathcal{B}_{u p} & \mathcal{B}_{u u}
\end{array}\right)=T_{2}^{-1}, \\
& Z_{2}=\frac{\tilde{p}_{2}}{\tilde{u}_{2}}=\frac{\mathcal{C}_{p u}}{\left(1-\mathcal{C}_{u u}\right)\left(1-\mathcal{C}_{p p}\right)-\mathcal{C}_{p u} \mathcal{C}_{u p}},
\end{aligned}
$$

and

$$
\left(\begin{array}{ll}
\mathcal{C}_{p p} & \mathcal{C}_{p u} \\
\mathcal{C}_{u p} & \mathcal{C}_{u u}
\end{array}\right)=\mathbf{T}_{\mathbf{T C}} \cdot \mathbf{T}_{\mathbf{1}}
$$

Therefore, assuming that $\tilde{p}_{0}=P_{0}$ at position $x=0$ one gets $\tilde{u}_{0}=P_{0} / Z_{0}$, and

TABLE III. Performances for a standing-wave engine equipped with a ceramic catalyst stack $\left(L_{1}=160 \mathrm{~cm}\right.$ and $\left.L_{2}=10 \mathrm{~cm}\right)$.

\begin{tabular}{lrc}
\hline \hline & Gain $G$ & Operating frequency $f_{\text {op }}$ \\
\hline$Q_{H}=9 \mathrm{~W}$ & -0.14 & $79.7 \mathrm{~Hz}$ \\
$Q_{H}=18 \mathrm{~W}$ & 0.91 & $81.1 \mathrm{~Hz}$ \\
$Q_{H}=27 \mathrm{~W}$ & 1.50 & $82.1 \mathrm{~Hz}$ \\
$Q_{H}=36 \mathrm{~W}$ & 1.82 & $83.2 \mathrm{~Hz}$ \\
\hline \hline
\end{tabular}

$$
\begin{aligned}
& \left(\begin{array}{c}
\tilde{p}_{2} \\
\tilde{u}_{2}
\end{array}\right)=\mathbf{T}_{2} \cdot\left(\begin{array}{c}
\tilde{p}_{0} \\
\tilde{u}_{0}
\end{array}\right), \\
& \left(\begin{array}{c}
\tilde{p}_{1} \\
\tilde{u}_{1}
\end{array}\right)=\left(\begin{array}{cc}
1 & 0 \\
0 & \frac{1-\mathcal{C}_{p p}}{\mathcal{C}_{p u}}
\end{array}\right)\left(\begin{array}{c}
\tilde{p}_{2} \\
\tilde{u}_{2}
\end{array}\right), \\
& \left(\begin{array}{c}
\tilde{p}_{l} \\
\tilde{u}_{l}
\end{array}\right)=\mathbf{T}_{\mathbf{1}} \cdot\left(\begin{array}{c}
\tilde{p}_{1} \\
\tilde{u}_{1}
\end{array}\right), \\
& \left(\begin{array}{c}
\tilde{p}_{r} \\
\tilde{u}_{r}
\end{array}\right)=\mathbf{T}_{\mathbf{T A C}} \cdot\left(\begin{array}{c}
\tilde{p}_{l} \\
\tilde{u}_{l}
\end{array}\right) .
\end{aligned}
$$

From these relations, one gets the acoustic work produced within the TAC [Eq. (19)] and the acoustic work

$$
\begin{aligned}
\mathbf{W}_{\text {loss }}= & {\left[\frac{1}{2} \Re\left(\tilde{p}_{2} \tilde{u}_{2}^{*}\right)-\frac{1}{2} \Re\left(\tilde{p}_{0} \tilde{u}_{0}^{*}\right)\right] } \\
& +\left[\frac{1}{2} \Re\left(\tilde{p}_{l} \tilde{u}_{l}^{*}\right)-\frac{1}{2} \Re\left(\tilde{p}_{1} \tilde{u}_{1}^{*}\right)\right]
\end{aligned}
$$

consumed in the remaining of the device, which leads to the expression of the thermoacoustic amplification gain $G=\mathbf{W}_{\text {TAC }} /\left|\mathbf{W}_{\text {loss }}\right|$.

\section{B. Calculation of $G$ and $f_{o p}$. from experimental data}

As soon as the experimental transfer matrix of a given thermoacoustic core is measured for various heating conditions, an optimization of the thermoacoustic amplification gain can be done thanks to the theory given in the previous section. This optimization is achieved for a given heat power supply by searching the geometry of the thermoacoustic device which leads to a maximum value for $G$. In our case study, this amounts to determining the optimal lengths $L_{1}$ and $L_{2}$ within their respective ranges, which are for instance bounded by the frequency limits, i.e., from 30 to $500 \mathrm{~Hz}$ in our measurements. However, for illustration purposes, we chose to show $G$ and $f_{\text {op }}$. for a narrower interval, from 30 to $250 \mathrm{~Hz}$, as the optimal $G$ is still embraced in all cases. This optimization is done for each one of the four thermoacoustic cores made of different materials.

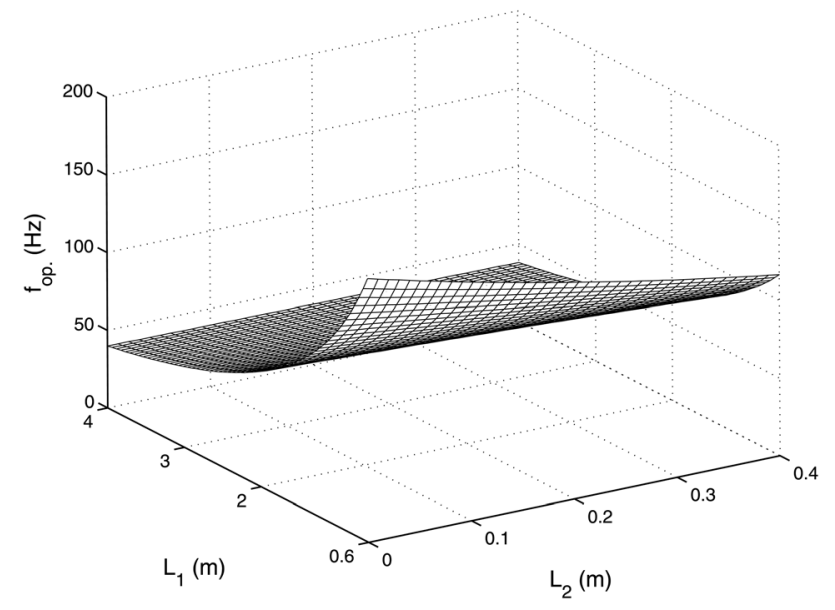

FIG. 9. $f_{\text {op. }}$ for standing-wave engines at $Q_{H}=81 \mathrm{~W}$ for the Ceramic Catalyst. 


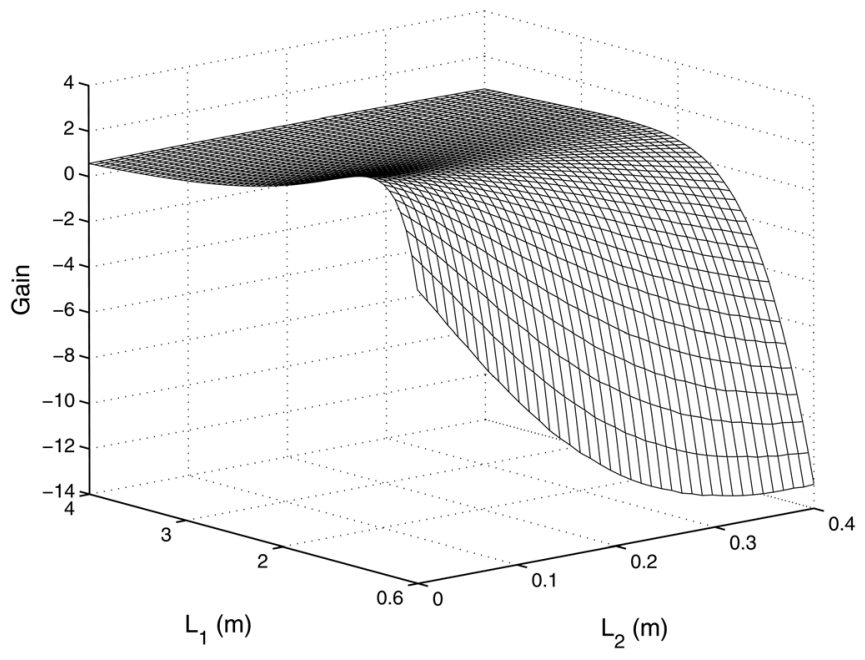

FIG. 10. Gain for standing-wave engines at $Q_{H}=81 \mathrm{~W}$ for the Ceramic Catalyst.

\section{Standing-wave engines}

For the standing-wave configuration, optimization results are given in Figs. 9 and 10 for the case of the ceramic stack and for the maximum value of heat supply $Q_{H}=81 \mathrm{~W}$. Figure 9 shows the evolution of the operating frequency $f_{o p}$., and Fig. 10 shows the corresponding thermoacoustic amplification gain $G$ as a function of the variable lengths $0.6 \mathrm{~m}$ $\leq L_{1} \leq 4 \mathrm{~m}$ and $0 \mathrm{~m} \leq L_{2} \leq 0.4 \mathrm{~m}$. Note that for each configuration (in terms of $L_{1}$ and $L_{2}$ ), the operating frequency $f_{o p}$. is searched around the first resonance mode (although higher order modes might become more unstable for some values of the lengths $L_{1}$ and $L_{2}$ ). A peak value of $G=3.31$ is reached with $L_{1}=1.06 \mathrm{~m}$ and $L_{2}=0 \mathrm{~m}$, at $f_{o p}=121 \mathrm{~Hz}$. It is worth mentioning that the obtained value for the "optimum" length $L_{2}$ equals 0 , which is equivalent to closing the right side of the TAC by a rigid plug: this result suggests that the length of Thermal Buffer Tube of the TAC under test is too long (in the sense that if a shorter TBT had been used, a non-zero optimal length $L_{2}$ might have been expected).

The same analysis is done for the other 3 materials, and results are summarized in Table IV. None of them reaches the thermoacoustic outbreak, as their optimal gain are below the unity. In the case of the Nichrome Foam, the maximum $G$ is not so smaller than 1 , which is consistent with this material geometrical properties (see Table I), closer to the ones of the Ceramic Catalyst; one may expect to reach the onset when slightly increasing $Q_{H}$. In the case of the RVC Foam the results are less promising than the Ceramic Catalyst's case. However, it is worth noting that the maximum heat supply for the RVC Foam is only $18 \mathrm{~W}$ due to its temperature limitation in the presence of oxygen (combustion hazard): such a

TABLE IV. Maximal performances and respective configurations for standing-wave engines at $Q_{H}=81 \mathrm{~W}$.

\begin{tabular}{lrccc}
\hline \hline & $G$ & $f_{\text {op. }}(\mathrm{Hz})$ & $L_{1}(\mathrm{~m})$ & $L_{2}(\mathrm{~m})$ \\
\hline Ceramic Catalyst & 3.31 & 121 & 1.06 & 0 \\
Stainless Steel Grids & -0.42 & 30.2 & 5.50 & 0 \\
RVC Foam $\left(Q_{H}=18 \mathrm{~W}\right)$ & -0.14 & 33.7 & 4.75 & 0 \\
NiCr Foam & 0.84 & 81.8 & 1.85 & 0 \\
\hline \hline
\end{tabular}

TABLE V. Maximal performances and respective configurations for closedloop engines at $Q_{H}=81 \mathrm{~W}$.

\begin{tabular}{lcccc}
\hline \hline & $G$ & $f_{\text {op. }}(\mathrm{Hz})$ & $L_{1}(\mathrm{~m})$ & $L_{2}(\mathrm{~m})$ \\
\hline Ceramic Catalyst & 1.47 & 156 & 0 & 0.77 \\
Stainless Steel Grids & 0.46 & 55.1 & 0 & 2.68 \\
RVC Foam $\left(Q_{H}=18 \mathrm{~W}\right)$ & 0.87 & 42.1 & 0.49 & 3.24 \\
NiCr Foam & 6.88 & 35.2 & 0.90 & 3.80 \\
\hline \hline
\end{tabular}

material should be possibly employed as a stack if an inert gas were chosen as the working fluid. At last, as what would be expected, the pile of stainless steel grids is the worst material of all for the standing-wave engine; it has the lowest porosity and smaller average pore radius, which are undesirable aspects for a standing-wave system. These 4 materials have best performance with $L_{2}=0$. For both RVC Foam and Stainless Steel Grids the $f_{o p}$. results not much higher than $30 \mathrm{~Hz}$, with $L_{1}$ at the upper limit of the established constraints, what compels a less well defined $G$ peak for these general conditions and frequency range.

\section{Closed-loop engines}

The same kind of comparative analysis is led for the closed-loop configuration. The results are summarized in Table V. For this configuration, the pile of stainless steel grids is expected to lead to the best result. Nevertheless, despite of its favorable properties mentioned before, such material appears in the last performance position. However, when noting the typical lengths of alike materials usually employed as regenerators, which are around few centimeters, it becomes suggestive to consider our $7-\mathrm{cm}$ pile as too long. Indeed, this conclusion would be in agreement with the strong decay of acoustic pressure level along its axis, as verified during our measurements of its $\mathbf{T}_{\mathrm{TAC}}$. Hence, besides low porosity and small average pore radius, this pile also exhibits a length too large, which contributes proportionally to the viscous losses.

Actually, the best performing material here tested is the Nichrome Foam, whose maximum gain reaches 6.88 at $35.2 \mathrm{~Hz}$, with $L_{1}=0.90 \mathrm{~m}$ and $L_{2}=3.80 \mathrm{~m}$, as illustrated in Figs. 11 and 12 (note that a contour plot is preferred to a

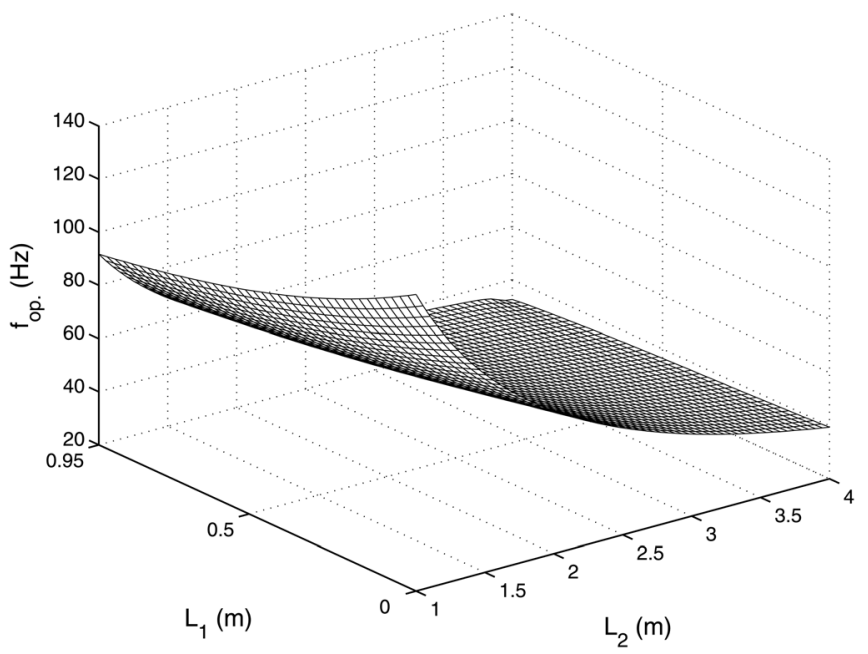

FIG. 11. $f_{o p}$. for closed-loop engines at $Q_{H}=81 \mathrm{~W}$ for the Nichrome Foam. 


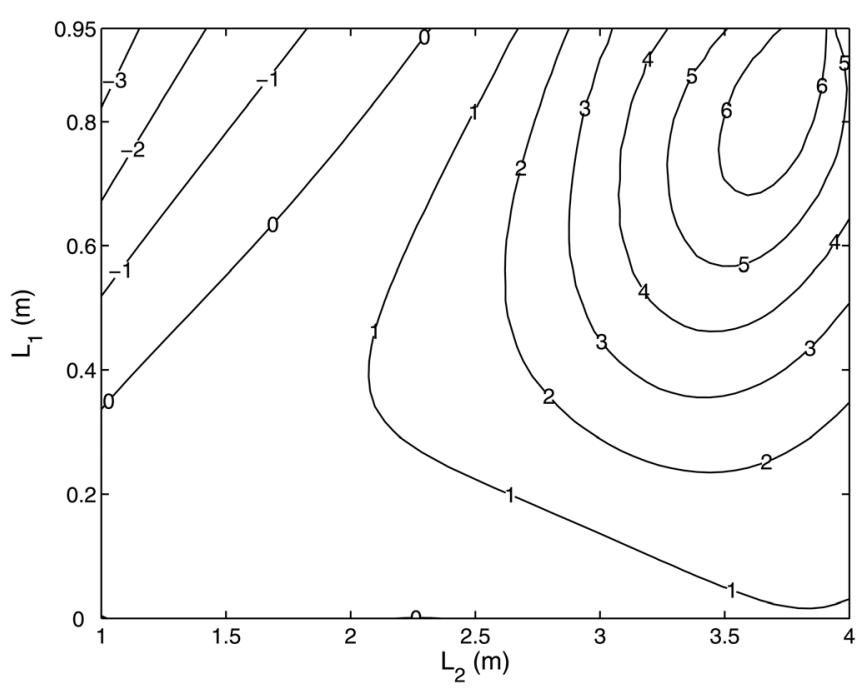

FIG. 12. Gain for closed-loop engines at $Q_{H}=81 \mathrm{~W}$ for the Nichrome Foam.

surface area in Fig. 12 which allows to observe more clearly the optimum values of $L_{1}$ and $L_{2}$ ). From the knowledge of the operating frequency, $f_{o p}=35.2 \mathrm{~Hz}$, it is quite direct to estimate the associated thermal boundary layer thickness at room temperature $\delta_{\kappa} \approx 450 \mu \mathrm{m}$, which is almost as high as the average pore radius ( $600 \mu \mathrm{m}$, see Table I) of the sample.

The Ceramic Catalyst is the second material that performs above $G=1$ for this engine, as shown in Table V and Fig. 13, which is the contour plot of the thermoacoustic gain $G$ as a function of $L_{1}$ and $L_{2}$. The maximum gain $G$ is 1.47 with a corresponding frequency $f_{o p}=156 \mathrm{~Hz}$. In that specific case, the process of optimization leads to an engine which is different from the one obtained with the NiCr Foam. The estimate of the thermal boundary layer thickness at $158 \mathrm{~Hz}$ indeed leads to $\delta_{\kappa} \approx 200 \mu \mathrm{m}$ which is lower than the average pore radius (530 $\mu \mathrm{m}$, calculated from data in Table I): the obtained engine is thus much more a closed-loop but stack-based engine rather than a regenerator-based thermoacoustic Stirling engine.

The RVC Foam does not reach the thermoacoustic outbreak in this engine, but its maximum heat supply $(18 \mathrm{~W})$ is

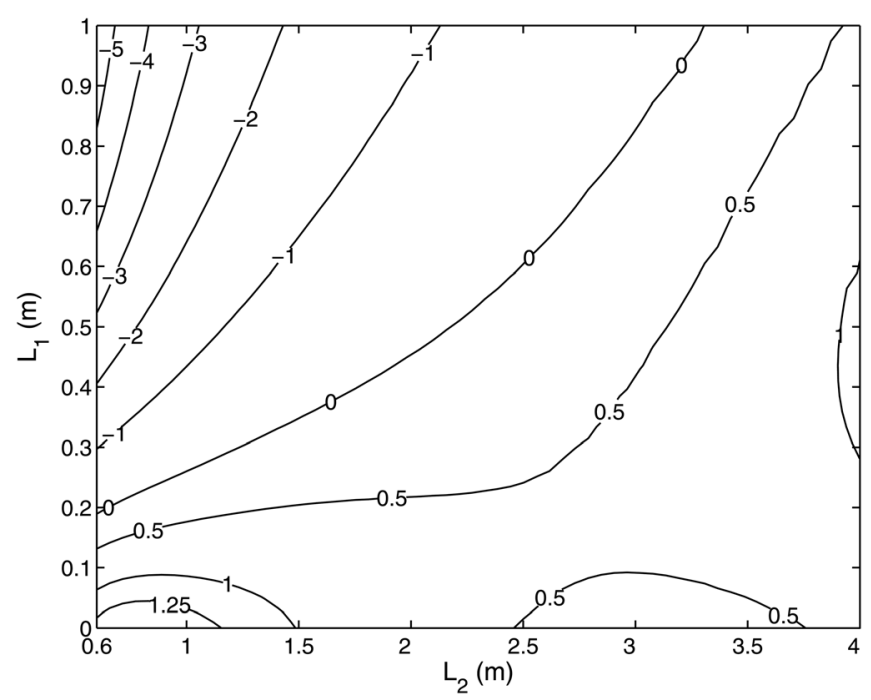

FIG. 13. Gain for closed-loop engines at $Q_{H}=81 \mathrm{~W}$ for the Ceramic Catalyst.
4.5 smaller than others maximums $(81 \mathrm{~W})$. Considering that even though with a small $Q_{H}$ the optimal $G$ reaches 0.87 , one may guess that its gain would overcome the unity easily before $(81 \mathrm{~W})$. An experiment in the absence of oxygen could verify the validity of this statement.

\section{DISCUSSION AND CONCLUSION}

We have presented an alternative method for the transfer matrix measurement of a thermoacoustic core. This method has been proved to be accurate, and such accuracy could be verified because of the precision of the acoustic impedance sensor, to the care taken in its calibration, and to the close monitoring over the variation of the temperature distribution. We have shown that this method, contrarily to the two-load method, is accomplishing independently on the material under test, even if dealing with one that resembles a lengthy regenerator.

The T-matrix components of the TAC have been measured for different kinds of materials under different heating conditions, and these data have been used to calculate the associated operating frequency and thermoacoustic amplification gain in both a simple standing-wave engine and a simple closed-loop engine. An optimization process of the engine's operation has been proposed, which consists of maximizing the thermoacoustic amplification gain, and the results obtained also give the opportunity to compare the performances of different materials. The optimization method presented in Sec. III B could be applied to more complicated engines as the one depicted in Fig. 1, with much more parameters to adjust (notably the electromechanical parameters of the linear alternator). However, in what concerns the engine performance evaluation, the works presented in this paper also reveal important features to be improved later on, in future experiments, aiming at more reliable results.

The low predicted performance for the closed-loop engine with the Stainless Steel Grids indicates that this sample may be too long (at least if it shall be used as a regenerator) so that it is difficult to apply a steep temperature gradient along it in order to reach a substantial thermoacoustic amplification, as it would be expected for such material; besides, the proportional viscous dissipation also contributes in this sense. These results are in accordance with the difficulties encountered during the transfer matrix measurements by the two-load method due to the strong acoustic pressure decay. These difficulties, for instance, have led to the development of the alternative method to overcome them. Therefore, the alternative method has been proved to be capable to evaluate a regenerator for a wide range of lengths (till at least $7-\mathrm{cm}$ ) with enough accuracy to allow an engine performance prediction. The performance prediction also allows the evaluation of the outreach limits of the TBT length. In the case of the Ceramic Catalyst for the standingwave engine, for example, the TBT length seems too high, as it is suggested that one would find a better performance for a shorter TBT and a non-zero optimal length $L_{2}$.

For practical reasons, air at atmospheric pressure was chosen as the working fluid, despite of being inappropriate from the performance concerns (most engines make use of 
pressurized helium). Therefore, due to this, the predicted onset of thermoacoustic instability $(G>1)$ was not reached for all kind of materials, and therefore, no formal comparative analysis could be achieved.

The results obtained in Sec. III B have not been confirmed by experiments, notably for the reasons mentioned above but also for practical aspects relative to the difficulty in building the complete engine (for instance, the closedloop engine equipped with a ceramic catalyst stack is impossible to be built unless bending the TAC). This kind of experimental confirmation has already been carried on by Guedra et al., ${ }^{19}$ and it is anticipated that it should also succeed here (at least for the prediction of threshold) since the modeling of Sec. III B is nothing else than the propagation of plane acoustic waves in lossy ducts.

It is worth mentioning that the works presented here are intrinsically limited to the acoustic pressure amplitudes for which the linear approximation can be retained. The measurement method of Sec. II could be, in principle, extended to large amplitudes but some nonlinear processes like the generation of acoustic streaming could not be adequately characterized by this way. Actually, the thermoacoustic amplification gain $G$ should be considered cautiously as soon as $G>1$. The physical meaning of a gain significantly larger than unity (e.g., $G=6.88$ in Table V) is that the heated TAC under consideration has a very high potential of thermoacoustic amplification, but it is clear that as soon as self-sustained oscillations are generated, the associated heat transport by sound (with subsequent variations of the Tmatrix components) should be considered.

${ }^{1}$ G. W. Swift, Thermoacoustics-A Unifying Perspective for Some Engine and Refrigerators (Acoustical Society of America, Melville, NY, 2002), $300 \mathrm{pp}$.

${ }^{2} \mathrm{P}$. Ceperley, "A pistonless Stirling engine-The traveling wave heat engine," J. Acoust. Soc. Am. 66(5), 1508-1513 (1979).

${ }^{3}$ S. Backhaus and G. W. Swift, "A thermoacoustic Stirling heat engine," Nature 399, 335-338 (1999).

${ }^{4}$ W. C. Ward and G. W. Swift, "Design environment for low-amplitude thermoacoustic engines," J. Acoust. Soc. Am. 95(6), 3671-3672 (1994).

${ }^{5}$ G. Penelet, S. Job, V. Gusev, P. Lotton, and M. Bruneau, "Dependence of sound amplification on temperature distribution in annular thermoacoustic engines," Acust. Acta Acust. 91(3), 567-577 (2005).

${ }^{6} \mathrm{H}$. Yuan, S. Karpov, and A. Prosperetti, "A simplified model for linear and nonlinear processes in thermoacoustic prime movers. Part 2: Nonlinear oscillations," J. Acoust. Soc. Am. 102(6), 3497-3506 (1997).

${ }^{7}$ S. Karpov and A. Prosperetti, "A nonlinear model of thermoacoustic devices,” J. Acoust. Soc. Am. 112(4), 1431-1444 (2002).
${ }^{8}$ M. F. Hamilton, Y. A. Ilinskii, and E. A. Zabolotskaya, "Nonlinear twodimensional model for thermoacoustic engines," J. Acoust. Soc. Am. 111(5), 2076-2086 (2002).

${ }^{9}$ G. Penelet, V. Gusev, P. Lotton, and M. Bruneau, "Experimental and theoretical study of processes leading to steady-state sound in annular thermoacoustic engines," Phys. Rev. E 72(1), 016625 (2005).

${ }^{10}$ H.-S. Roh, W. P. Arnott, J. M. Sabatier, and R. Raspet, "Measurement and calculation of acoustic propagation constants in arrays of small air-filled rectangular tubes," J. Acoust. Soc. Am. 89(6), 2617-2624 (1991).

${ }^{11}$ G. W. Swift and R. M. Keolian, "Thermoacoustics in pin-array stacks," J. Acoust. Soc. Am. 94(2), 941-943 (1993).

${ }^{12}$ L. A. Wilen, "Measurements of thermoacoustic functions for single pores,” J. Acoust. Soc. Am. 103(3), 1406-1412 (1998).

${ }^{13}$ L. A. Wilen, "Dynamic measurements of the thermal dissipation function of reticulated vitreous carbon," J. Acoust. Soc. Am. 109(1), 179-184 (2001).

${ }^{14}$ A. Petculescu and L. A. Wilen, "Lumped-element technique for the measurement of complex density," J. Acoust. Soc. Am. 110(4), 1950-1957 (2001).

${ }^{15}$ W. P. Arnott, H. E. Bass, and R. Raspet, "General formulation of thermoacoustics for stacks having arbitrarily shaped pore cross sections," J. Acoust. Soc. Am. 90(6), 3228-3237 (1991).

${ }^{16}$ J. A. Adeff, T. J. Hofler, A. A. Atchley, and W. C. Moss, "Measurements with reticulated vitreous carbon stacks in thermoacoustic prime movers and refrigerators," J. Acoust. Soc. Am. 104(1), 32-38 (1998).

${ }^{17} \mathrm{G}$. Petculescu and L. A. Wilen, "Thermoacoustics in a single pore with an applied temperature gradient," J. Acoust. Soc. Am. 106(2), 688-694 (1999).

${ }^{18}$ H. Roh, R. Raspet, and H. E. Bass, "Parallel capillary-tube-based extension of thermoacoustic theory for random porous media," J. Acoust. Soc. Am. 121(3), 1413-1422 (2007).

${ }^{19}$ M. Guedra, G. Penelet, P. Lotton, and J.-P. Dalmont, "Theoretical prediction of the onset of thermoacoustic instability from the experimental transfer matrix of a thermoacoustic core," J. Acoust. Soc. Am. 130(1), 145-152 (2011).

${ }^{20} \mathrm{H}$. Boden and M. Abom, "Influence of errors on the two-microphone method for measuring acoustic properties in ducts," J. Acoust. Soc. Am 79(2), 541-549 (1986).

${ }^{21}$ M. L. Munjal, Acoustics of Ducts and Mufflers With Application to Exhaust and Ventilation System Design (Wiley, New York, 1987), 352 pp.

${ }^{22}$ H. Hatori, T. Biwa, and T. Yazaki, "How to build a loaded thermoacoustic engine," J. Appl. Phys. 111, 074905 (2012).

${ }^{23}$ J.-P. Dalmont, "Acoustic impedance measurement, Part I: A review," J. Sound Vib. 243(3), 427-439 (2001).

${ }^{24}$ J.-P. Dalmont, "Acoustic impedance measurement, Part II: A new calibration method," J. Sound Vib. 243(3), 441-459 (2001).

${ }^{25}$ J. C. Le Roux, M. Pachebat, and J. P. Dalmont, "A new impedance sensor for industrial applications," in Acoustics 2012, Nantes, France (April 23-27, 2012)

${ }^{26} \mathrm{C}$. A. Macaluso and J. P. Dalmont, "Trumpet with near-perfect harmonicity: Design and acoustic results," J. Acoust. Soc. Am. 129(1), 404-414 (2011).

${ }^{27}$ A. A. Atchley, H. E. Bass, T. J. Hofler, and H. Lin, "Study of a thermoacoustic prime-mover below onset of self-oscillations," J. Acoust. Soc. Am. 91(2), 734-743 (1994).

${ }^{28}$ A. A. Atchley, "Analysis of the initial buildup of oscillations in a thermoacoustic prime-mover,” J. Acoust. Soc. Am. 95(3), 1661-1664 (1994). 